\title{
Saponins enhance exon skipping of 2'-O-methyl phosphorothioate oligonucleotide in vitro and
} in vivo

\author{
Mingxing Wang \\ Bo Wu \\ Sapana N Shah \\ Peijuan Lu \\ Qilong Lu
}

McColl-Lockwood Laboratory for Muscular Dystrophy Research, Department of Neurology, Cannon Research Center, Carolinas Medical Center, Charlotte, NC 28203, USA

Correspondence: Mingxing Wang McColl-Lockwood Laboratory for Muscular Dystrophy Research,

Department of Neurology, Cannon Research Center, Carolinas Medical Center, 1000 Blythe Blvd, Charlotte, NC 28203, USA

Tel +l 7043555588

Fax +I 7043551679

Email mingxing.wang@atriumhealth.org
This article was published in the following Dove Press journal:

Drug Design, Development and Therapy

Background: Antisense oligonucleotide (ASO)-mediated exon skipping has been feasible and promising approach for treating Duchenne muscular dystrophy (DMD) in preclinical and clinical trials, but its therapeutic applications remain challenges due to inefficient delivery.

Methods: We investigated a few Saponins for their potential to improve delivery performance of an antisense 2'-Omethyl phosphorothioate RNA (2'-OMePS) in muscle cells and in dystrophic $m d x$ mice. This study was carried out by evaluating these Saponins' toxicity, cellular uptake, transduction efficiency in vitro, and local delivery in vivo for 2'-OMePS, as well as affinity study between Saponin and 2'-OMePS.

Results: The results showed that these Saponins, especially Digitonin and Tomatine, enhance the delivery of 2'-OMePS with comparable efficiency to Lipofectamine $2 \mathrm{k}$ (LF-2k) -mediated delivery in vitro. Significant performance was further observed in $m d x$ mice, up to 10-fold with the Digitonin as compared to 2'-OMePS alone. Cytotoxicity of the Digitonin and Glycyrrhizin was much lower than LF-2k in vitro and not clearly detected in vivo under the tested concentrations.

Conclusion: This study potentiates Saponins as delivery vehicle for 2'-OMePS in vivo for treating DMD or other diseases.

Keywords: antisense delivery, 2'-OMePS, exon skipping, saponin, muscular dystrophy

\section{Introduction}

Duchenne muscular dystrophy (DMD) is an X-linked recessive disease caused by nonsense or frameshift mutation in the dystrophin gene mainly affecting boys at a rate of 1 in every 3,500 live births. ${ }^{1-3}$ Fundamental treatments of DMD requires either correction or replacement of the mutated gene to restore function. The dystrophin gene is one of the largest genetic loci and contains at least 79 exons distributed over 2.5 million base pair on the p21 subregion of $\mathrm{X}$ chromosome, and it is an essential component of the dystrophin glycoprotein complex, which links the actin cytoskeleton to the extracellular matrix to provide sarcolemma stability during muscle contraction and coordinates signal transducers at the sarcolemma. The large size of dystrophin protein and the requirement of lifetime administration still severely challenge the progress in developing effective clinical therapies. ${ }^{4}$ Antisense oligonucleotide (ASO)-mediated exon skipping has been demonstrated to be promising for the treatment of DMD; this strategy aims to intentionally skip one or multiple exons to restore the normal reading frame of the mutated transcripts, which results in the production of internally truncated but functional dystrophin proteins. ${ }^{5-16}$ ASOs are short, single-stranded sequences of synthetic and chemically modified RNA or DNA, which are capable of hybridizing specific targets by the Watson-Crick rules. Among the several ASO chemistries developed for the 
exon-skipping strategy, only $2^{\prime}$-O-methyl-phosphorothioate RNA (2'-OMePS) and phosphorodiamidate morpholino (PMO) have been tested in clinical trials for the treatment of DMD and other neuromuscular diseases. Oligomers of the 2 '-OMePS backbone are resistant to nuclease degradation and relatively stable in biological systems compared with natural DNA or RNA, but the negatively charged phosphorothioate linkage might reduce binding affinity and increase toxicity, which has limited the ceiling of dosage for clinical trials. ${ }^{17,18}$ These issues taken together suggest that an important challenge that remains is to improve the delivery of $2^{\prime}$-OMePS to the targeted tissues safely. ${ }^{12,19,20}$ Studies in animal models have demonstrated that a significant therapeutic effect of ASO on DMD can be achieved through the following: 1) Polymer-based nonviral delivery strategy. 2'-OMePS in combination with the PEG550-PEI2k copolymer enhanced exon skipping in skeletal muscles, but the hydrophilic nature of the polymer hinders the interactions between the polymer with 2 -OMePS and cell/tissue membranes, leading to limited effect. ${ }^{21,22}$ Applying this approach, we have developed a series of amphiphilic cationic polymers, and the results demonstrated that the polymer's size, hydrophilic-lipophilic balance (HLB), and composition are key factors for improving ASO's delivery efficiency. ${ }^{23-27} 2$ ) Chemical modification with tissue-homing peptides has been reported with significant heightening in targeting dystrophin exons and in the levels of dystrophin expression in $m d x$ mice. However, the complicated synthesis and purification increase the cost, and peptiderelated potential immune responses might prevent repeated administration. ${ }^{28,29}$ 3) Small molecules-aided approach, which have been demonstrated to enhance exon skipping of ASOs in $m d x$ mice. For example, Dantrolene enhanced antisensemediated exon skipping in vitro and in vivo as reported by Kendall et al, ${ }^{30}$ and the monosaccharide-formulated ASOs enhanced delivery efficiency with high safety margin as studied by Han et al group. ${ }^{31,32}$ Although some promising results have been achieved by the above strategies, the development of an efficient and safe delivery approach still remains as one of the main challenges of turning 2'-OMePS into a significant therapeutic outcome for the treatment of DMD.

Recently, we investigated a few saponins for PMO delivery in muscle cells and in vivo and observed the dramatical enhancement of the targeted dystrophin exon 23 skipping in $m d x$ mice. Digitonin (D) increases exon skipping up to sevenfold compared with PMO alone. ${ }^{33}$ The results indicate that the amphiphilic nature of saponins is the key beneficial factor for forming stable complexes with uncharged oligonucleotide PMOs and enables it to pass through the hydrophobic membrane. Based on the abovementioned encouraging results, we report herein the study of saponins for negatively charged $2^{\prime}$-OMePS delivery in vitro and in dystrophic $m d x$ mice.

The delivery performance of 2'-OMePS in vitro and in vivo was studied by formulation with saponins - a class of natural amphiphile composed of hydrophilic glycone and hydrophobic aglycone, commonly found in a set of plants and are important nutrition for human and animals. Various saponin-rich extracts have been demonstrated with health benefits on blood cholesterol levels, cancer, and bone health (http://www.phytochemicals.info/phytochemicals/ saponins.php). The amphiphilic nature, immunological role, and divergent biological activities have made saponins a suitable adjuvant for drug delivery. ${ }^{34,35}$ However, few attempts have been made to examine saponins as an oligonucleotide delivery vehicle. Considering the use of saponin in vaccines and our recently reported study on PMO delivery, ${ }^{33,35,36}$ we hypothesized that saponin may act as a neutral, biocompatible vector for negative antisense 2 '-OMePS delivery in the treatment of muscular dystrophy.

In view of the safety and cost, as a delivery vehicle, we investigated four saponins which are commercially available and have been widely applied as biomaterials (Figure 1). They are D, Glycyrrhizin (G), Tomatine (T), and Lanoxin (L). The first three were examined in our previous PMO delivery study. ${ }^{33}$ 1) $\mathrm{D}$ - a steroidal saponin (saraponin) obtained from Digitalis purpurea. Its aglycone is digitogenin (a spirostan steroid) having several potential membranerelated applications in biochemistry. ${ }^{37,38}$ 2) $\mathrm{G}$ - provides the main sweet flavor for Glycyrrhiza glabra (liquorice), with potential immunomodulating, anti-inflammatory, hepato- and neuro-protective effects. Its aglycone has been used as a prodrug to prevent liver carcinogenesis in chronic hepatitis C patients. ${ }^{39,40} 3$ ) $\mathrm{L}$ - also named Digoxin obtained from Digitalis lanata. ${ }^{41,42}$ It is in the WHO List of Essential Medicines and is used to treat various heart conditions ( $\underline{\mathrm{htp}} \mathrm{://}$ www.who.int/medicines/publications/essentialmedicines/ en/). 4) T - a glycoalkaloid found mainly in the stems and leaves of tomato plants. It has fungicidal, antimicrobial, and insecticidal properties, and the related aglycon, tomatidine, has multiple health benefits. ${ }^{43,44}$ The study on saponins for the delivery of $2^{\prime}$-OMePS in cell culture and in vivo in the $m d x$ mice are described herein for the first time.

\section{Materials and methods Materials}

DMEM, FBS, penicillin-streptomycin, 4-(2-hydroxyethyl)1-piperazineethanesulfonic acid buffer solution (1M), and 


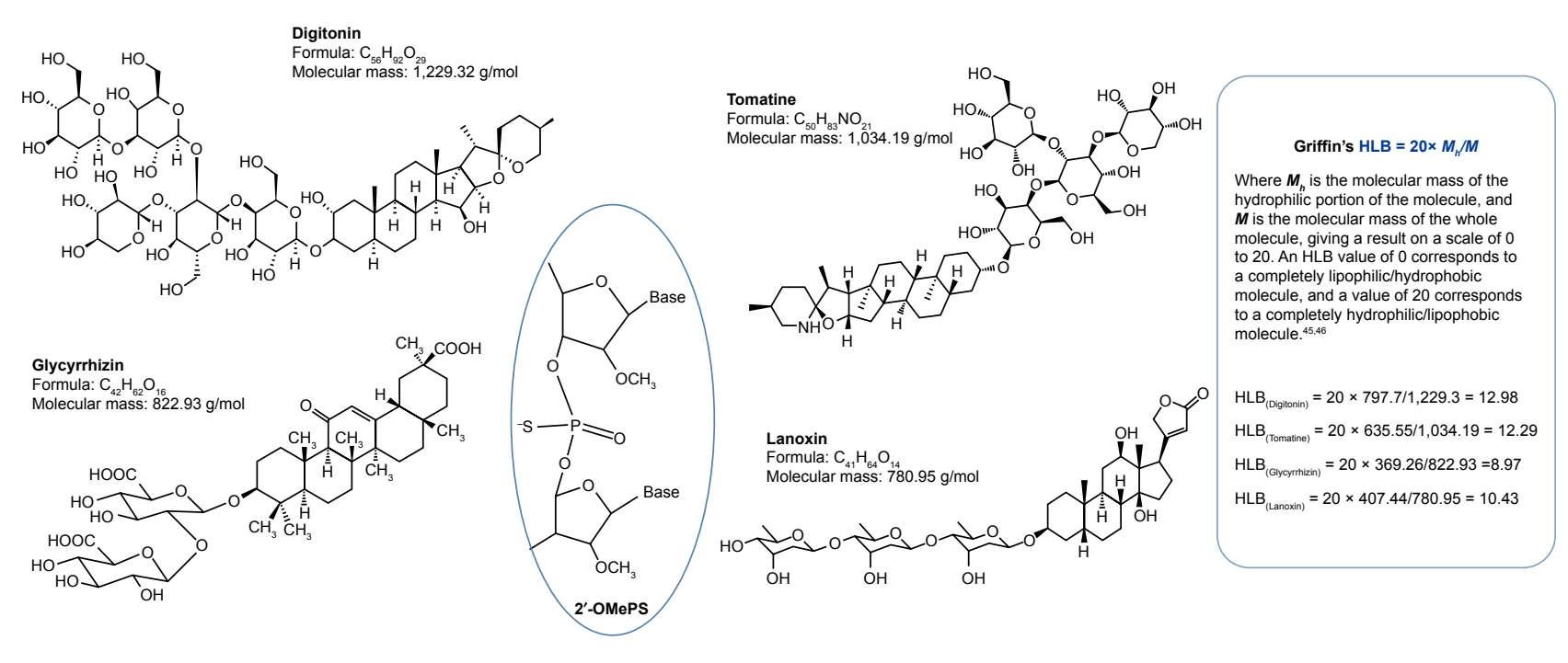

Figure I Chemical structures of saponins, 2'-OMePS, and relative HLB of saponins. Abbreviations: HLB, hydrophilic-lipophilic balance; 2'-OMePS, 2'-O-methyl phosphorothioate RNA.

L-glutamine were ordered from Thermo Fisher Scientific (Waltham, MA, USA). 3-(4,5-Dimethylthiazol-2-yl)-5(3-carboxymethoxyphenyl)-2-(4-sulfophenyl)-2H-tetrazolium (MTS) was bought from BioVision Inc. (Milpitas, CA, USA). ASOs modified by 2'-O-methylation and phosphorthioation 2'-OMePSE50 (27-mer, -19 to +8$)\left(5^{\prime}\right.$-AAC UUCCUCUUUAACAGAAAAGCAUAC- $3^{\prime}$ ) targeting the human dystrophin gene exon 50, 2'-OMePSE23 (20-mer, +2 to -18) (5'-GGCCAAACCUCGGCUUACCU-3') targeting the mouse dystrophin gene exon 23 were commercially purchased from GenScript ${ }^{\circledR}$ (Piscataway, NJ, USA). Saponins and all other chemicals were ordered from Sigma-Aldrich Co. (St Louis, MO, USA), unless otherwise stated. Investigated saponins' structures are illustrated in Figure 1.

\section{Cell lines}

C2C12 myoblast of mouse muscle was purchased from American Type Culture Collection (ATCC) (Manassas, VA, USA). C2C12E50 cell line expressed a human dystrophin exon 50 green fluorescent protein (hDysE50/ GFP)-based reporter. The construction of the hDysE50/ GFP-reporter vector was based on the procedure reported previously. ${ }^{47,48}$

The hDysE50 flanked by $600 \mathrm{bp}$ of its respective intronic sequences (intron 49 on the $5^{\prime}$ side and intron 50 on the $3^{\prime}$ side) was amplified from DNA of human myoblasts. The sequence was inserted into the middle of the $\beta$-globin intron sequence that was placed inside the coding sequence of GFP gene under the control of actin promotor.

\section{Cell viability assay}

Cytotoxicity was evaluated in $\mathrm{C} 2 \mathrm{C} 12$ cell line by MTSbased assay. Cells were seeded in a 96-well tissue culture plate at $1 \times 10^{4}$ cells per well in $200 \mu \mathrm{L} 10 \%$ FBS-DMEM. Cells achieving $70 \%-80 \%$ confluence were exposed to saponins at different doses, and incubated for 24 hours followed by addition of $20 \mu \mathrm{L}$ of CellTiter $96^{\circledR} \mathrm{AQ}$ ueous One solution proliferation kit (Promega Corporation, Fitchburg, WI, USA). After further incubation for 4 hours, the resultant water-soluble formazan color was read at $570 \mathrm{~nm}$ using a Tecan 500 Plate reader (Tecan US, Inc., Morrisville, NC, USA) to obtain the metabolic activity of the cell. Untreated cells were taken as controls with $100 \%$ viability and wells without cells as blanks; and the relative cell viability was calculated using the formula: $\left(A_{\text {treated }}-A_{\text {background }}\right) \times 100 /$ $\left(A_{\text {control }}-A_{\text {background }}\right)$. All viability assays were carried out in triplicate. ${ }^{25-27}$

\section{In vitro transfection}

The C2C12E50 myoblast cell line expressing a hDysE50/ GFP-based reporter was adapted in this study. ${ }^{47,48}$ The cells were seeded in a 24-well plate at a density of $5 \times 10^{4}$ cells/well and cultured in 10\% FBS-DMEM in a humidified incubator at $37^{\circ} \mathrm{C}\left(10 \% \mathrm{CO}_{2}\right)$. Once the cell grows to a $70 \%$ confluence, the culture medium was replaced 2 hours before the addition of saponin/2'-OMePSE50 (fixed at $5 \mu \mathrm{g}$ ) formulation with varying ratio. Lipofectamine $2 \mathrm{k}$ (LF-2k) was used as the control delivery vehicle. Transfection efficiencies indicated by GFP expression were initially visualized under a fluorescent microscope (Olympus IX71; 
Olympus America Inc., Centre Valley, PA, USA) after 3-day incubation. Transfection efficiency was further examined quantitatively using flow cytometry. Cells were washed twice with PBS (1X, pH 7.4) and released from culture vessel with $0.2 \mathrm{~mL} 0.05 \%$ trypsin-EDTA, neutralized by FBS, pelleted by centrifugation, and then resuspended in $0.5 \mathrm{~mL}$ of ice-cold PBS (1X, pH 7.4). Samples were run on a fluorescence-activated cell sorting (FACS) Calibur flow cytometer (BD, Franklin Lakes, NJ, USA). At least $1 \times 10^{4}$ cells were counted and analyzed with CellQuest Pro software package (BD).

\section{Cellular uptake and intracellular localization}

For cellular uptake and intracellular localization study, $\mathrm{C} 2 \mathrm{C} 12$ cells were seeded onto 12 -well plate at $5 \times 10^{4}$ cells/ well, and cultured to $50 \%$ confluence before the addition of saponin-formulated fluorescence-labeled 2'-OMePS complex at the predetermined ratio for testing. After 24 hours of addition of the samples, cells were washed with warm PBS to remove any residual saponin/oligonucleotide formulations not taken up by cells and incubated with media containing Lysotracker ${ }^{\circledR}$ Red DND-99 (Thermo Fisher Scientific) as per manufacturer's recommendation to label lysosomes. Cells were also counterstained with Hoechst 33342 (Thermo Fisher Scientific) to label cellular nuclei. Microscopy analysis was performed under the Olympus IX71 fluorescent microscope, and the resulting images for colocalization of saponin/2'OMePS to the lysosome was visualized by merged channel images.

\section{In vivo delivery}

This study was carried out in strict accordance with the recommendations in Guide for the Care and Use of Laboratory Animals of the National Institutes of Health, and the experiment protocols were approved by the Institutional Animal Care and Use Committee, Carolinas Medical Center (breeding protocol: 10-13-07A; experimental protocol: 10-13-08A).

\section{Animals and injections}

Mice were housed with 12-hour light-dark cycles in individually ventilated cages and had access to standard chow and water ad libitum. Dystrophic $m d x$ mice (C57BL/10 as genetic background) aged 4-5 weeks were used for in vivo testing (five mice per group [mixed gender in the test and control groups] unless otherwise stated). All injections were performed under isoflurane anesthesia, and all efforts were made to minimize suffering. ${ }^{13,14,47}$ The 2 '-OMePSE23 targeting the boundary sequences of exon and intron 23 of mouse dystrophin gene was used. For intramuscular (i.m.) injections, $5 \mu \mathrm{g}$ 2'-OMePSE23 with or without saponin was formulated in $40 \mu \mathrm{L}$ saline for each tibialis anterior (TA) muscle. The muscles were examined 2 weeks later.

\section{Immunohistochemistry and histology}

Serial sections of $6 \mu \mathrm{m}$ were cut from the treated mouse TA muscles, together with the control muscles. The sections were stained with a rabbit polyclonal antibody $\mathrm{P} 7$ for the detection of dystrophin protein as described previously. ${ }^{13,15}$ Polyclonal antibodies were detected by goat anti-rabbit IgG Alexa 594 (Thermo Fisher Scientific). The number of dystrophin-positive fibers in one section was addressed using the Olympus BX51 fluorescent microscope (Olympus America Inc.).

\section{Reverse transcription polymerase chain reaction (RT-PCR) and Western blot}

Total RNA was extracted from the muscle after dissection; $100 \mathrm{ng}$ of RNA template was used for a $25-\mu \mathrm{L}$ RT-PCR with the FideliTaq RT-MasterMix (USB, Cleveland, OH, USA). ${ }^{13,15}$ The primer sequences for the RT-PCR were Ex20Fo 5'-CAGAATTCTGCCAATTGCTGAG-3' and Ex26Ro 5'-TTCTTCAGCTTGTGTCATCC-3' for amplification of mRNA from exons 20-26. The products were examined by electrophoresis on a $1.5 \%$ agarose gel. The intensity of the bands was measured with the NIH ImageJ 1.42 (National Institute of Health, Bethesda, MD, USA) and percentage of exon skipping was calculated with the intensity of the two bands representing both exon 23 unskipped and skipped as $100 \%$. Unskipped band with exon 23 is $1,093 \mathrm{bp}$, and skipped band without exon 23 is $880 \mathrm{bp}$. Protein extraction and Western blot were performed as described previously. ${ }^{13,15}$ The membrane was probed with NCL-DYS1 monoclonal antibody against dystrophin rod domain (1:200 dilutions; Vector Laboratories, Burlingame, CA, USA). The bound primary antibody was detected by horseradish peroxidase-conjugated goat anti-mouse IgG (1:3,000 dilutions; Santa Cruz Biotechnology Inc., Dallas, TX, USA) and the ECL Western Blotting Analysis System (PerkinElmer Inc., Waltham, MA, USA). The intensity of the bands obtained from the treated $m d x$ mice muscles was measured with ImageJ 1.42 software and compared with that of normal muscles of C57BL/6 mice. $\alpha$-Actin was detected by rabbit anti-actin antibody (Sigma-Aldrich Co.) as a sample loading control. 


\section{UV-Vis study}

The absorbance of saponin formulated 2'-OMePS was determined with NanoDrop 2000 spectrophotometer (Thermo Fisher Scientific). A total of $1.5-\mu \mathrm{L}$ of each sample was measured at desired concentration under room temperature.

\section{Transmission electron microscopy}

The saponin/2'-OMePS complex solution containing $1 \mu \mathrm{g}$ of $2^{\prime}$-OMePS was prepared at different weight ratios of saponin/2'-OMePS in $100 \mu \mathrm{L} 0.9 \%$ saline and analyzed using transmission electron microscopy (TEM) with AMTXR80S-B wide angle side Mount 8M Pixel, CCD Camera (JEM-1400Plus Transmission Electron Microscope by JEOL USA, Inc., Peabody, MA, USA). The samples were prepared using negative staining with $1 \%$ phosphotungstic acid. ${ }^{33}$

\section{Statistical analysis}

All the results were expressed as mean $\pm \mathrm{SEM}$, and two-tailed Student's $t$-test with a value of $* P \leq 0.05$ was considered statistically significant.

\section{Results and discussion Cytotoxicity studies}

The cytotoxicity of the saponins was evaluated in $\mathrm{C} 2 \mathrm{C} 12$ cells by MTS-based assay under different concentrations (from 2 to $100 \mu \mathrm{g} / \mathrm{mL}$ ) as shown in Figure 2. Toxicity varied from high to low with T (Mw: 1,034.19, HLB: 12.29), L (Mw: 780.95, HLB: 10.43), D (Mw: 1,229.32, HLB: 12.98), to G (Mw: 822.93, HLB: 8.97), which is in line with the behavior in $\mathrm{C} 2 \mathrm{C} 12 \mathrm{E} 50$ cell line for $\mathrm{D}, \mathrm{G}$, and $\mathrm{T} \cdot{ }^{33}$ The cell viability was $85.3 \%, 75.4 \%, 30.8 \%, 26.9 \%$, and $18.4 \%$ at the dose of $20 \mu \mathrm{g} / \mathrm{mL}$ for G, D, L, T, and LF-2k, respectively. Cells with $\mathrm{G}$ treatment even at the highest dose of $100 \mu \mathrm{g} / \mathrm{mL}$ showed limited toxicity with about $90 \%$ cells remaining alive. The low toxicity of $\mathrm{G}$ is probably due to its relative smaller molecule and its aglycone part, although it is more hydrophobic than the other three counterparts. The high toxicity of $\mathrm{T}$ is probably related to aglycone alkaloid, different from the $\mathrm{D}, \mathrm{G}$, and $\mathrm{L}$, and more hydrophobic than $\mathrm{D}$. These results demonstrated that these saponins especially $\mathrm{G}$ and $\mathrm{D}$ are much less toxic than LF-2k, the current commercial standard vector for $p$ DNA or negative-charged oligonucleotides delivered in vitro.

\section{Delivery of 2'-OMePS with saponins in vitro}

The exon-skipping efficacy of saponin-mediated ASO delivery in vitro was evaluated with $\mathrm{C} 2 \mathrm{C} 12 \mathrm{E} 50$ myoblasts, which can stably express a GFP reporter bifurcated by the insertion of the hDysE50.47,48 The expression of GFP in the reporter cells relies on the skip of exon 50 by ASOs. The 2'-OMePSE50 sequence ( $5^{\prime}$-AACUUCCUCUUUAACAGAAAAGCAUA C-3') targeting the inserted hDysE50 within the GFP coding region was used as usual. ${ }^{27} \mathrm{C} 2 \mathrm{C} 12 \mathrm{E} 50$ cells were treated with a fixed amount $(4 \mu \mathrm{g} / \mathrm{mL})$ of 2'-OMePSE50 formulated with each of the saponins at five escalating doses $(2,5,10$, 20 , and $50 \mu \mathrm{g}$ ), in total $500 \mu \mathrm{L} 10 \%$ FBS-DMEM medium. Transduction efficiency was visualized by a fluorescent

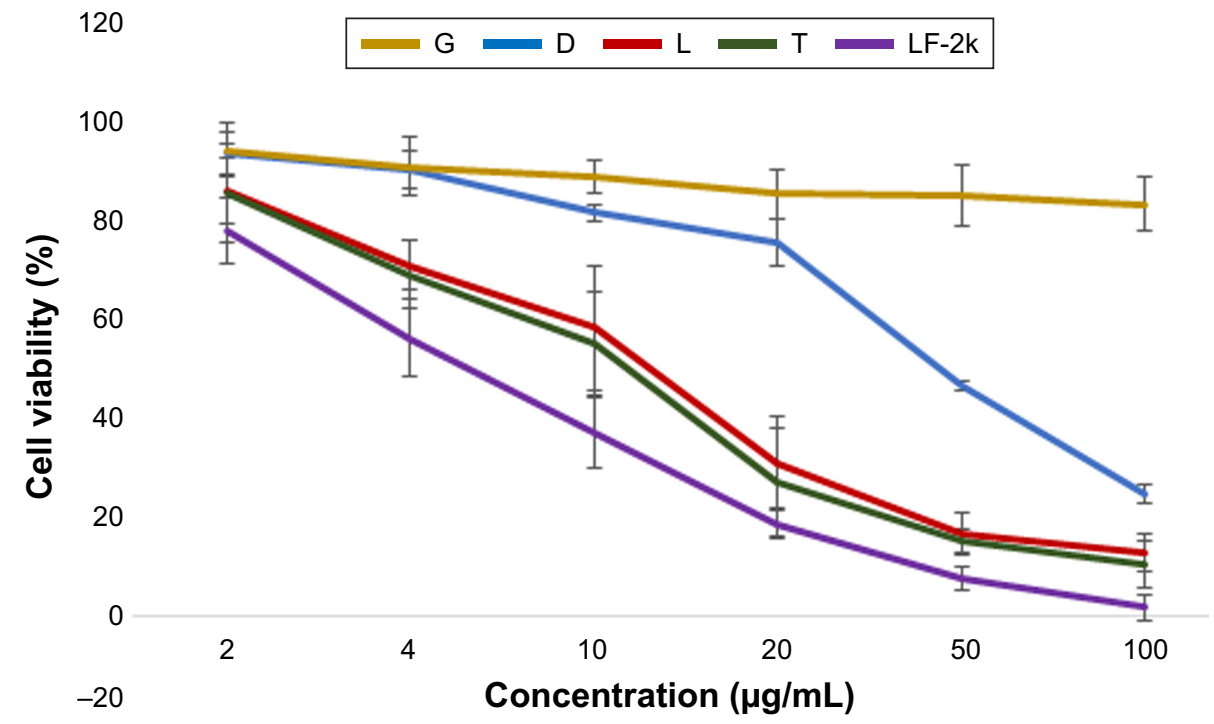

Figure 2 Cell viability of $\mathrm{C} 2 \mathrm{Cl} 2$ myoblasts determined by MTS assay when exposed to saponins with six doses $(2,4,10,20,50$, and I00 $\mu \mathrm{g} / \mathrm{mL}$; LF-2k as control). Notes: Cells were seeded in 96 -well plates at an initial density of $I \times 10^{4}$ cells/well in $0.2 \mathrm{~mL}$ growth media. The results are presented as the mean \pm SEM ( $\mathrm{n}=3$ ).

Abbreviations: D, Digitonin; G, Glycyrrhizin; L, Lanoxin; T, Tomatine; LF-2k, lipofectamine 2k; MTS, 3-(4,5-Dimethylthiazol-2-yl)-5-(3-carboxymethoxyphenyl)-2(4-sulfophenyl)-2H-tetrazolium. 
microscope and quantitively determined by FACS analysis after 3-day treatment (Figure 3). The results indicated that $\mathrm{D}(\mathrm{Mw}=1,229.32 \mathrm{~g} / \mathrm{mol})$ and $\mathrm{T}(\mathrm{Mw}=1,034.19 \mathrm{~g} / \mathrm{mol})$ at $2 \mu \mathrm{g}$ improved GFP expression significantly compared with 2 '-OMePS alone, better than or comparable to LF-2k, and the ceiling dose were 10 and $5 \mu \mathrm{g}$ for $\mathrm{D}$ and T reaching GFP expression of $76.5 \%$ and $39.4 \%$, respectively. The levels of GFP expression were dominantly saponin's structuredependent: the larger the molecule, the more effective they are as $2^{\prime}$-OMePS delivery vehicle, which is consistent with the results of our early report. ${ }^{27,33}$ While the smaller molecules $\mathrm{G}(\mathrm{Mw}=822.93 \mathrm{~g} / \mathrm{mol})$ with $21.5 \%$ at dose of $20 \mu \mathrm{g}$ and $\mathrm{L}(\mathrm{Mw}=780.95 \mathrm{~g} / \mathrm{mol})$ with $25.6 \%$ at the dose of $10 \mu \mathrm{g}$, demonstrate lower efficiency than $\mathrm{D}(\mathrm{Mw}=1,229.32 \mathrm{~g} / \mathrm{mol})$ and $\mathrm{T}(\mathrm{Mw}=1,034.19 \mathrm{~g} / \mathrm{mol})$, both are larger and more hydrophilic. In contrast, below $3 \%$ of the cells were GFP positive when treated by $2^{\prime}$-OMePS alone. The GFP expression produced from $\mathrm{D}$ - and T-mediated 2 -OMePS delivery was about 26, 13-fold higher than that for 2'-OMePS only at their optimum dosage. D's high efficiency is also likely attributed to its better permeabilizing cell membranes. ${ }^{37,38}$ The exon-skipping efficiency is obviously dose-dependent and toxicity related. This is clearly demonstrated with D and $\mathrm{T}$ at the doses higher than 10 and $5 \mu \mathrm{g}$, respectively, showing a sharp decline in both GFP expression and cell viability. Cell viability of saponin-formulated 2'-OMePS was like that of saponin only in $\mathrm{C} 2 \mathrm{C} 12$ cell line.

The transduction was further analyzed with cytotoxicity by staining with propidium iodide (PI) and Hoechst 33342 after 6-day transfection (Figure 4). The delivery efficiency of D-formulated 2'-OMePS was much higher than that of LF-2k-mediated one at the optimum condition based on our
A

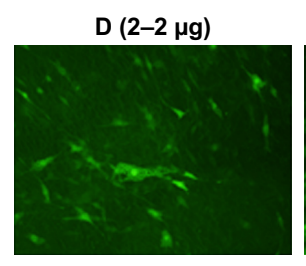

G (5-2 $\mu \mathrm{g})$

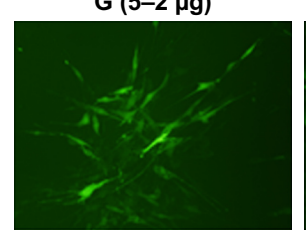

B

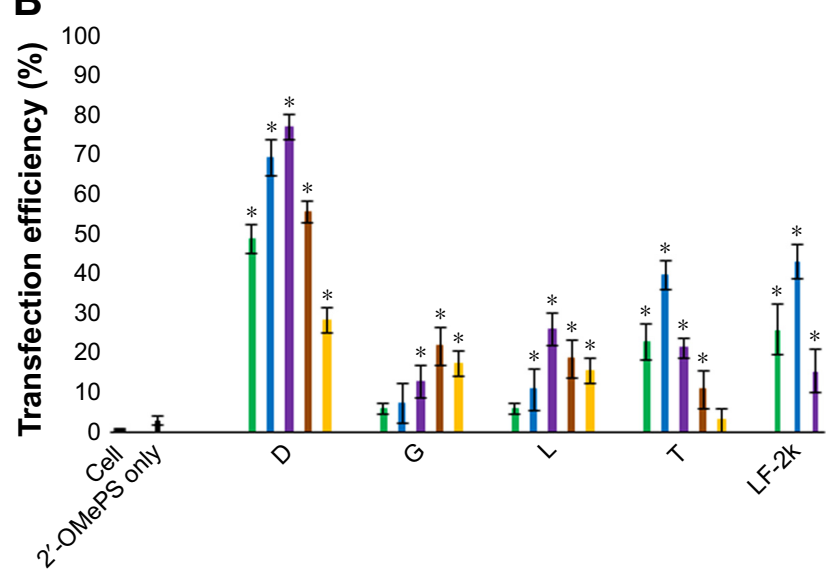

$D(10-2 \mu g)$

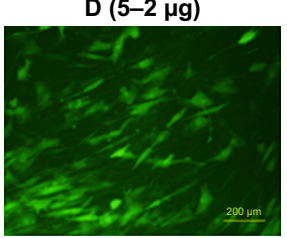

G (10-2 $\mu \mathrm{g})$

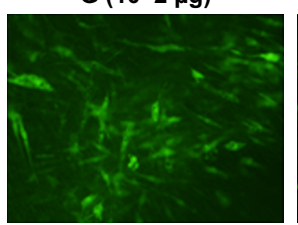

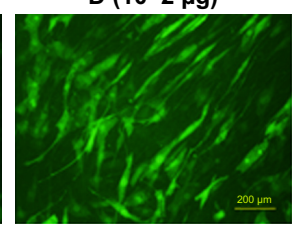

$\mathrm{T}(10-2 \mu \mathrm{g})$

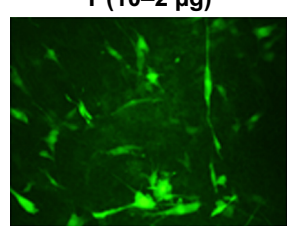

C
D $(20-2 \mu \mathrm{g})$

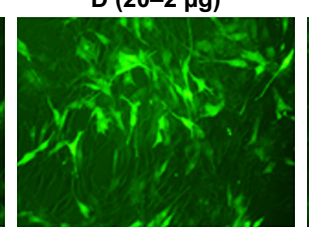

LF-2k (4-2 $\mu g)$

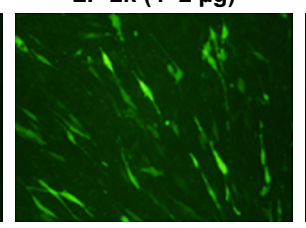

L (10-2 $\mu g)$

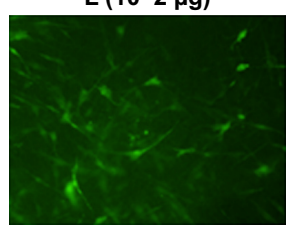

2'-OMePSE50 (2 $\mu \mathrm{g})$

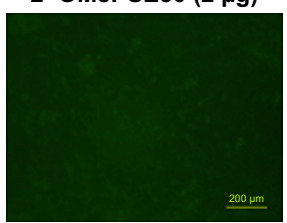

100

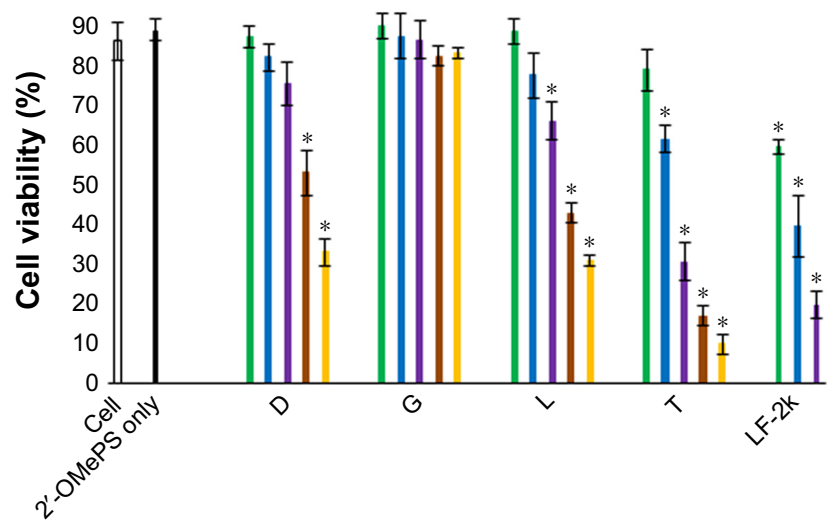

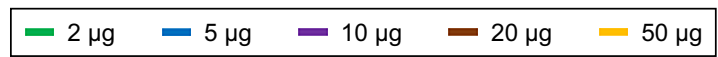

Figure 3 Delivery performances of 2'-OMePSE50/saponin complexes in $\mathrm{C} 2 \mathrm{Cl} 2 \mathrm{E} 50$ cell line.

Notes: (A) Fluorescence images of 2'-OMePS-induced reporter GFP expression with and without saponins for exon 50 skipping. The images were taken after 3-day treatment (original magnification, $\times 200$; scale bar: $500 \mu \mathrm{m}$ ). (B) Transduction efficiency of $2^{\prime}$-OMePS formulated with saponins (two-tailed Student's $t$-test, $* P \leq 0.05$ compared to $2^{\prime}$-OMePS alone). (C) Cell viability (two-tailed Student's $t$-test, ${ }^{*} P \leq 0.05$ compared to untreated cell). In this test, 2 '-OMePSE50 (2 $\mu \mathrm{g}$ ) was formulated with each of the saponins $(2,5,10,20$, and $50 \mu \mathrm{g})$ and LF- $2 \mathrm{k}(2,5$, and $10 \mu \mathrm{g})$ formulated as control in $0.5 \mathrm{~mL} 10 \% \mathrm{FBS}-\mathrm{DMEM}$ medium. The results are presented as the mean \pm SEM in triplicate.

Abbreviations: D, Digitonin; G, Glycyrrhizin; L, Lanoxin; T, Tomatine; GFP, green fluorescent protein; LF-2k, Lipofectamine 2k; 2'-OMePS, 2'-O-methyl phosphorothioate RNA. 


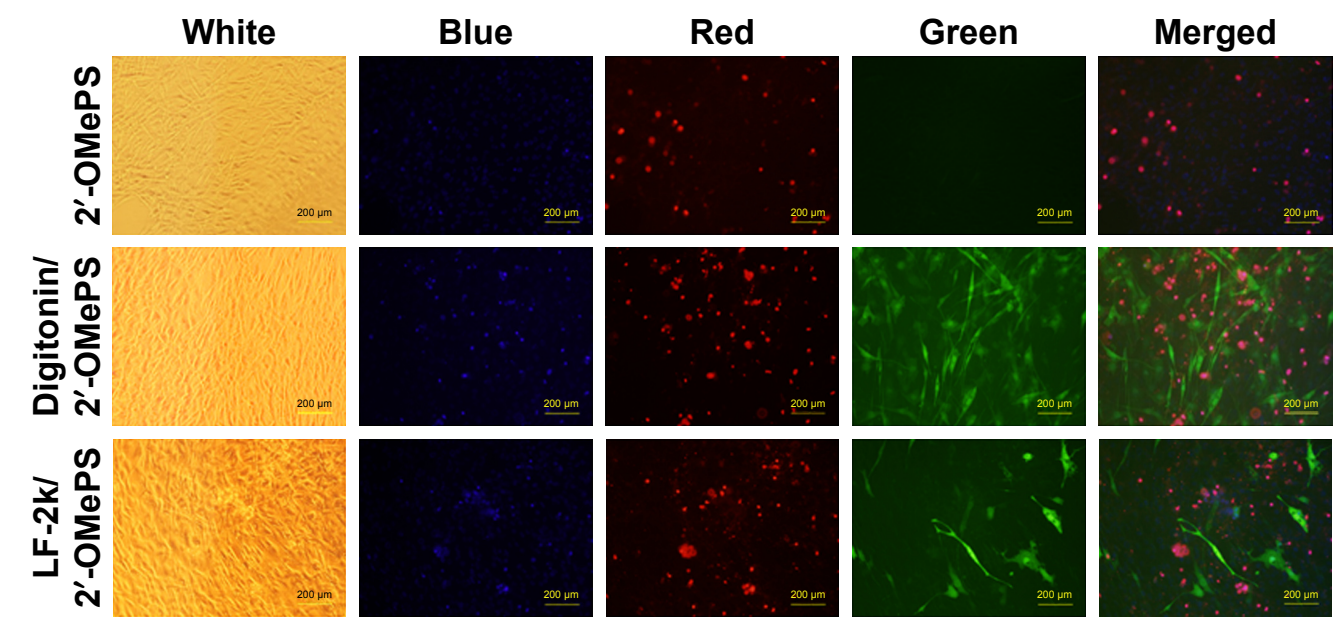

Figure 4 Comparison of transduction and cytotoxicity between D ( $10 \mu \mathrm{g})$ and LF-2k (4 $\mu \mathrm{g})$-mediated 2'-OMePSE50 (2 $\mu \mathrm{g})$ stained by PI (red) and Hoechst 33342 (blue) after 6-day transfection (original magnification, $\times 200$; scale bar: $500 \mu \mathrm{m})$.

Abbreviations: D, Digitonin; LF-2k, Lipofectamine 2k; 2'-OMePS, 2'-O-methyl phosphorothioate RNA; PI, propidium iodide.

previous study, ${ }^{26,27}$ while the toxicity was much lower than that of LF-2k; even D's dose was 2.5 times to that of LF-2k. This further indicates the advantage of saponin as a carrier of oligonucleotide delivery against LF-2k, which is probably due to saponin's amphiphilicity and neutral charge.

\section{Cellular uptake and intracellular localization}

To study the intracellular localization of saponin/2'-OMePS complex, D was complexed with fluorescein isothiocyanate (FITC) labeled 2'-OMePS at a weight ratio of 5:1. The presence of D apparently affected the cellular uptake of oligonucleotide 2 -OMePS as demonstrated by microscopy analysis (Figure 5). Signals for 2'-OMePS were considerably stronger and colocalized with lysosome and some enter the nuclei of $\mathrm{C} 2 \mathrm{C} 12$ cells when formulated with $\mathrm{D}$ than that of $2^{\prime}$-OMePS alone, which indicated the formation of a compacted $\mathrm{D} / 2^{\prime}$-OMePS complex, and release of oligonucleotide from the complex. Therefore, saponin showed its ability to efficiently deliver oligonucleotide $2^{\prime}$-OMePS, which might attribute to D's permeabilization of cell membranes. ${ }^{38}$

\section{Delivery of 2'-OMePS with saponins in vivo}

We next evaluated the effect of the saponins on $2^{\prime}$-OMePS delivery in vivo by i.m. injection in $m d x$ mice. The mouse model contains a nonsense mutation in exon 23, preventing the production of functional dystrophin protein. 2'-OMePSE23 targeting dystrophin exon 23 was injected into TA muscle for skipping the mutated exon 23 , thus restoring the reading frame of dystrophin transcripts and expression of truncated dystrophin protein. In view of the delivery performance in vitro, we chose $20 \mu \mathrm{g}$ saponins as effective and safe dosage and premixed it with $5 \mu \mathrm{g}$ of $2^{\prime}$-OMePS in $40 \mu \mathrm{L}$ saline for i.m. injection. The same amount of $2^{\prime}$-OMePS alone was used as control.

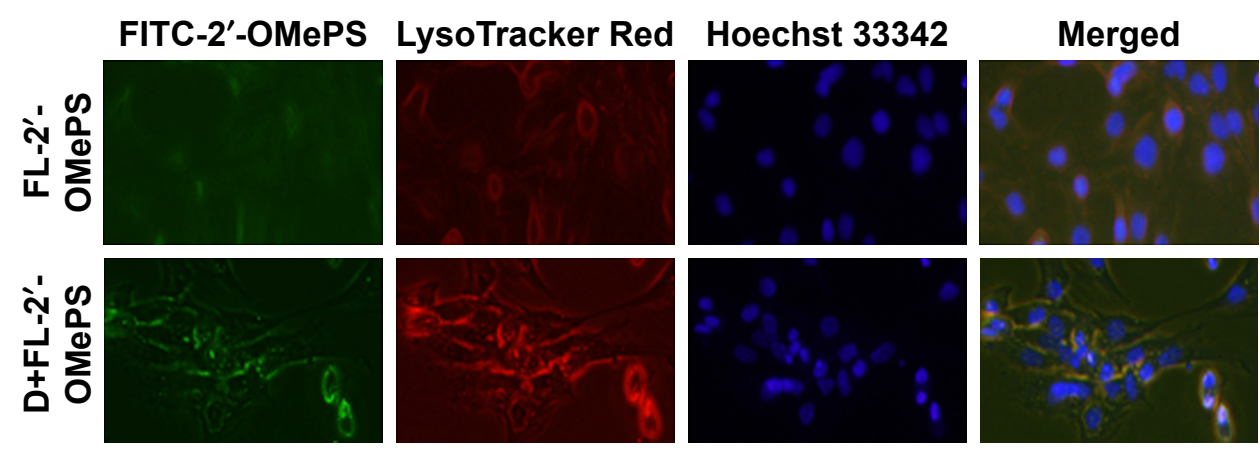

Figure 5 Microscopic images of 2'-OMePS-treated $\mathrm{C} 2 \mathrm{Cl} 2$ cells with and without D after 24 hours delivery. 2'-OMePS (green), lysosomes (red), and nuclei (blue) were illustrated with FITC-labeled 2'-OMePS, LysoTracker Red, and Hoechst 33342, respectively.

Abbreviations: D, Digitonin; FITC, fluorescein isothiocyanate; 2'-OMePS, 2'-O-methyl phosphorothioate RNA. 
Immunohistochemistry showed that mice treated with saponin-formulated 2'-OMePSE23 dramatically increased the number of dystrophin-positive fibers, reaching up to $25 \%$, $18 \%, 15 \%$, and $20 \%$ in one cross section of the TA muscle for D-, G-, L-, and T-vectored 2'-OMePS, respectively, in comparison with only $2.5 \%$ positive fibers with $2^{\prime}$-OMePS only. $\mathrm{D}$ showed the highest efficiency with nearly ten-fold over the control 2'-OMePS. Meanwhile, the levels of exon skipping and corresponding dystrophin expression were further quantitatively examined by RT-PCR and Western blot. D-, G-, L-, and T-formulated 2 '-OMePS and control 2'-OMePS achieved the levels of exon skipping at $38.6 \%, 29.1 \%, 28.9 \%, 27.5 \%$, and $6.3 \%$, respectively, and dystrophin protein expression at levels of $29.5 \%, 10.2 \%, 11.3 \%, 32.8 \%$, and $10.6 \%$ (C57 normalized as $100 \%$ ), respectively (Figure 6). These results did not correlate well with the data in vitro, indicating the gap occurred between in vitro and in vivo performances, also implying the notion that the composition within the vector molecule are crucial to improve the delivery efficiency of therapeutic agent in vivo. These results demonstrated that: 1) the amphiphilic nature of saponins as delivery carrier is the key for oligonucleotide delivery, which has been indicated in the delivery for both negative-charged 2 '-OMePS and neutral PMO, even when the delivery efficiency for 2 -OMePS is lower than that for PMO, probably due to the uncharged or less positive nature of saponins in physiological condition. The hydrophobic interaction and potential H-bond between saponin and 2 -OMePS are likely able to condense and stabilize the saponin $/ 2^{\prime}$-OMePS complex, thus improving the delivery efficiency; ${ }^{33} 2$ ) the better delivery performances are also relative to the saponin's permeabilizing ability to cell membrane. Taken together, these results further highlight the complexity of the interaction between delivery carrier and their delivery cargos.

\section{Interaction between saponin and $2^{\prime}$-OMePS}

To understand how the saponins improve the delivery performance of oligonucleotide 2 -OMePS, the affinity between saponin and oligonucleotide was studied. We first examined the interactions between the saponin and 2'-OMePS at different weight ratios by UV-Vis spectrum: saponin G or

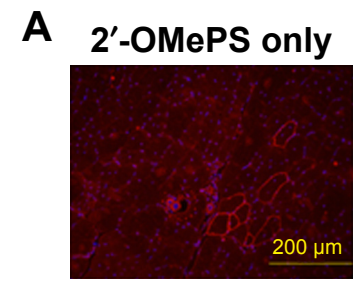

Blank

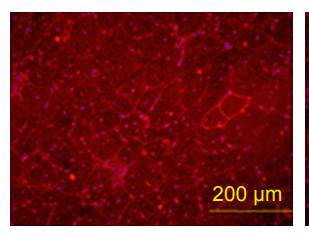

C

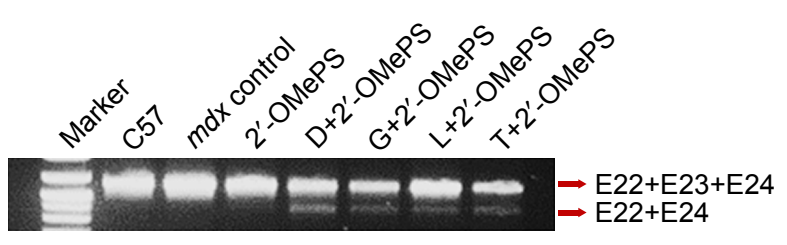

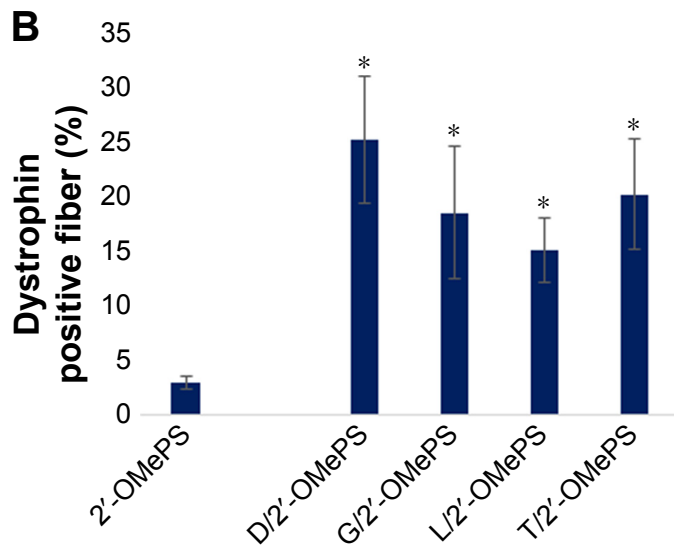

D

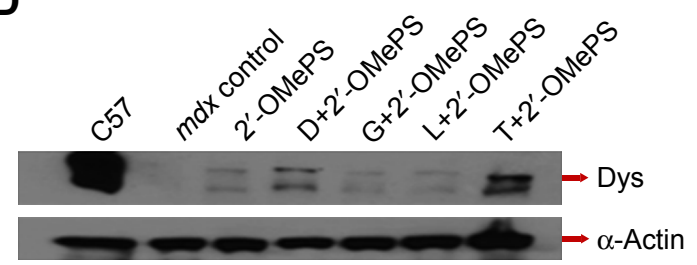

Figure 6 Dystrophin restoration in TA muscles of treated $m d x$ mice (aged 4-5 weeks) after i.m. injection for 2 weeks (the samples were from muscles treated with saponin [20 $\mu \mathrm{g}]$-formulated 2'-OMePSE23 [5 $\mu \mathrm{g}]$ in $40 \mu \mathrm{L}$ saline, and 2'-OMePSE23 only as control).

Notes: (A) Immunohistochemistry for dystrophin expression (original magnification, $\times 100$; scale bar: $200 \mu \mathrm{m}$ ). (B) The percentage of dystrophin-positive fibers counted from cross-section area of the muscles ( $n=5$, two-tailed $t$-test, $* P \leq 0.05$ compared with 2 -OMePS). (C) RT-PCR to detect exon-skipping efficiency. Total RNA of 100 ng from each sample was used for the amplification of dystrophin mRNA from exons 20-26. The upper bands (I,093 bp, indicated by E22+E23+E24) correspond to the normal mRNA, and the lower bands ( $880 \mathrm{bp}$, indicated by E22+E24) correspond to the mRNA with skipped exon E23. (D) Western blots demonstrate the expression of dystrophin protein from treated $m d x$ mice in comparison to C57BL/6 and untreated $m d x$ mice ( $10 \mu g$ of total protein was loaded for each sample). Dys, dystrophin detected with monoclonal antibody Dys I. $\alpha$-Actin was used as loading control.

Abbreviations: D, Digitonin; G, Glycyrrhizin; L, Lanoxin; T, Tomatine; i.m., intramuscular; 2'-OMePS, 2'-O-methyl phosphorothioate RNA; RT-PCR, reverse transcription polymerase chain reaction; TA, tibialis anterior. 
$\mathrm{D} / 2^{\prime}$-OMePS complex at the weight ratios from 1:1 to $25: 1$ was studied, because $\mathrm{G}$ molecule has absorbance at UV that can be easily examined against D, L, or T (Figure 7A). The G/PMO complexes showed similar absorbance as 2'-OMePS only at the ratio of $1: 1$, while showing a hypsochromic shift and hyperchromic effect compared to 2'-OMePS only with the increase of weigh ratios, which were attributed to the absorbance from excess of $\mathrm{G}$ itself. As for the $\mathrm{D} / 2^{\prime}$-OMePS complex at different ratios (Figure 7B), we can see the hypochromic effect compared to 2'-OMePS only as the ratio increases, except for the highest ratio of 25:1. There was a strong interaction between $\mathrm{D}$ and 2'-OMePS probably resulting from the hydrophilic interaction and H-bond. The hyperchromic effect was observed at the ratio 25:1, likely due to the particle aggregation from excess $\mathrm{D}$. A very similar absorbance of 2'-OMePS was observed only for D/L/T-2'OMePS complexes at the ratio of $10: 1$, while $\mathrm{G} / 2^{\prime}$-OMePS complex was significantly obtained with hypsochromic shift
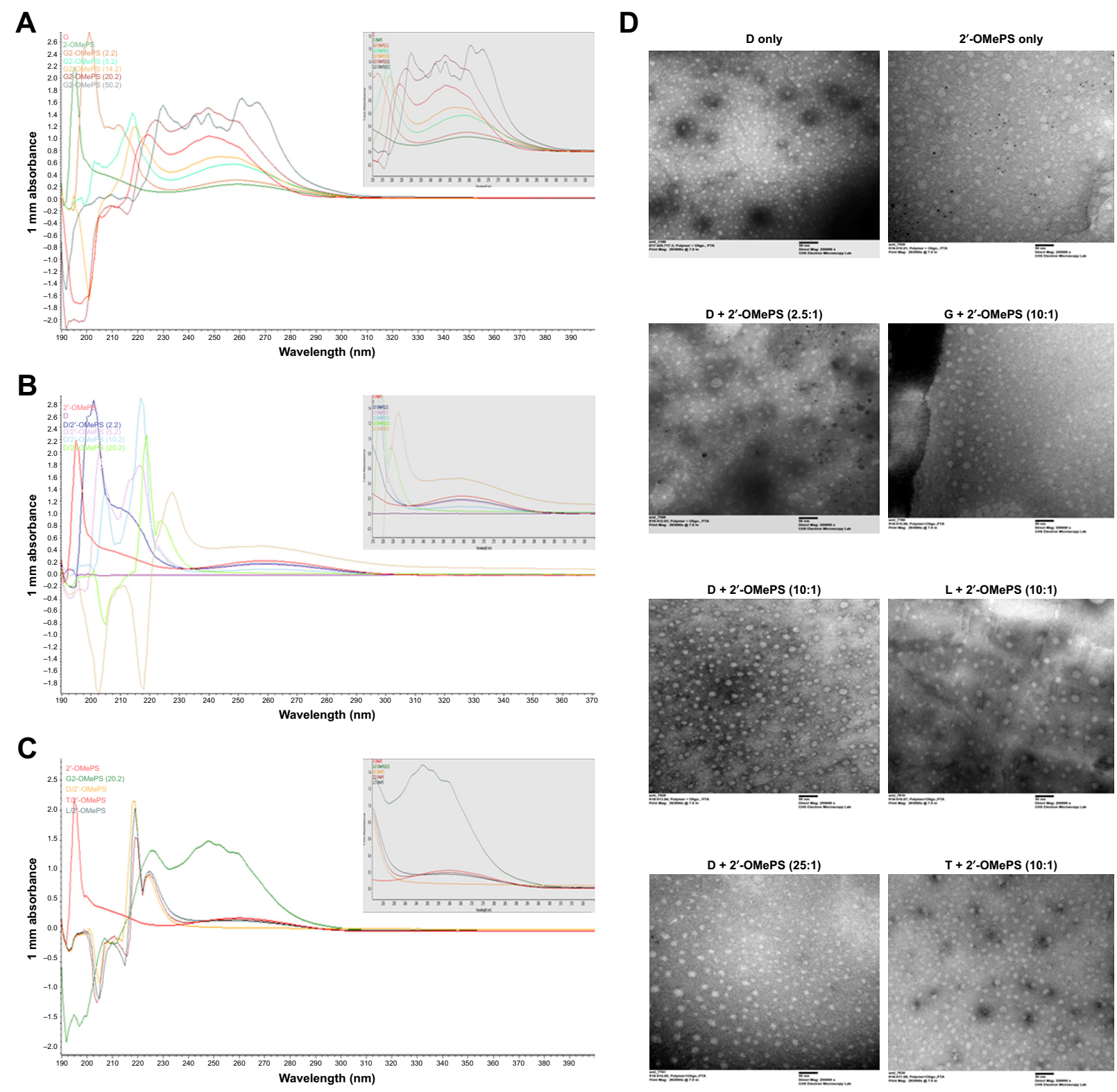

Figure 7 Affinity study between saponin and 2'-OMePS.

Notes: (A) UV-Vis of G-formulated 2'-OMePS at various weight ratios (I:I, 2.5:I, 5:I, I0:I, and 25:I). (B) UV-Vis of D-formulated 2'-OMePS at various weight ratios I:I, 2.5:I, 5:I, I0:I, and 25:I. (C) UV-Vis of D-, G-, L-, T-formulated 2'-OMePS at weight ratio 10:I (a total of $10 \mu \mathrm{L}$ in water, I.5 $\mu \mathrm{L}$ drop measured in NanoDrop 2000 at room temperature). (D) Negatively stained transmission electron micrographs (scale bar: $100 \mathrm{~nm}$ ) of D/G/L/T-formulated 2'-OMePS (I $\mu \mathrm{g})$ at different weight ratios in I00 $\mu \mathrm{L}$ $0.9 \%$ saline.

Abbreviations: D, Digitonin; G, Glycyrrhizin; L, Lanoxin; T, Tomatine; 2'-OMePS, 2'-O-methyl phosphorothioate RNA. 
and hyperchromic effect as compared to 2'-OMePS only (Figure 7C), which is related to G's absorbance against other three saponins without any absorbance over $220 \mathrm{~nm}$. The results indicated that the $\mathrm{D} / \mathrm{G} / \mathrm{L} / \mathrm{T}$ has similar affinity with oligomer 2'-OMePS, due to their shared amphiphilic nature and similar composition, although different in structure. Secondly, we further examined saponin/2'-OMePS complex at the weight ratio of $10: 1$ in $0.9 \%$ saline solution under TEM, and D/PMO at the ratio of 2.5:1 and 25:1 was also assessed. As illustrated in Figure 7D, the oligomer 2'-OMePS alone formed different size particles with some aggregation in the size below $10-50 \mathrm{~nm}$, which is likely resulted from the hydrophobic interaction and $\mathrm{H}$-bond in oligonucleotide itself; the saponin D formed uniform particles sized only below $15 \mathrm{~nm}$, while the D/2'-OMePS complexes showed larger particles around $10-30 \mathrm{~nm}$ at the ratios from $2.5: 1$ to 25:1, which were probably due to the strong hydrophobic interaction and H-bond as well as some consequent aggregation. The complex of saponin/2'-OMePS at the ratio of 10:1, the D/T-2'-OMePS complexes, produced clear uniform particles, probably due to the higher hydrophilic nature of $\mathrm{D} / \mathrm{T}$ relative to $\mathrm{G} / \mathrm{L}$; whereas $\mathrm{G} / \mathrm{L}-2^{\prime}$-OMePS complexes produced particles sized from 10 to $50 \mathrm{~nm}$, due to their more lipophilic nature compared with D or T and some aggregation. All the saponin/2'-OMePS complexes showed clear, separate, and small-sized particles compared with 2'-OMePS alone, which could be beneficial to their enhanced delivery performance. Clearly, detailed mechanisms of saponin to enhance 2 -OMePS delivery in vitro and in vivo remain to be further clarified.

\section{Conclusion}

In this study, some saponins have been evaluated as delivery vector for antisense 2 -OMePS both in vitro and in dystrophic $m d x$ mice. The results show that saponins, especially $\mathrm{D}$, improve the delivery with efficiency superior to LF-2kmediated delivery in vitro. A significant enhancement of $2^{\prime}$-OMePS delivery is further obtained in vivo, up to tenfold with $\mathrm{D}$ when compared to 2 -OMePS only. No obvious toxicity was observed in vivo at the tested dosage. $\mathrm{D}$ is the best delivery carrier that enables enhanced-skipping activity of both negative-charged 2'-OMePS and uncharged PMO, though the delivery efficiency of 2'-OMePS is slightly lower than that of PMO delivery both in vitro and in vivo. The data demonstrated the versatility of saponins as oligonucleotide delivery vehicle, and the optimization of molecular size and component has the potential toward a broad spectrum of therapeutic application.

\section{Acknowledgments}

We thank Dr David M Foureau and Dr Fei Guo for their technical assistance in flow cytometry and analysis; and Daisy M Ridings, Stephanie Williams, and Anthony Dart for their assistance with the Electron Microscopy Core Laboratory for the negative staining and TEM electron micrographs. This work was financially supported by the Carolinas Muscular Dystrophy Research Endowment at the Carolinas HealthCare Foundation and Carolinas Medical Center, Charlotte, NC.

\section{Disclosure}

The authors report no conflicts of interest in this work.

\section{References}

1. Emery AE. The muscular dystrophies. Lancet. 2002;359(9307): 687-695.

2. Hoffman EP, Brown RH, Kunkel LM. Dystrophin: the protein product of the Duchenne muscular dystrophy locus. Cell. 1987;51:919-928.

3. Mendell JR, Shilling C, Leslie ND, et al. Evidence-based path to newborn screening for Duchenne muscular dystrophy. Ann Neurol. 2012;71(3):304-313.

4. Long C, Amoasii L, Mireault AA, et al. Postnatal genome editing partially restores dystrophin expression in a mouse model of muscular dystrophy. Science. 2016;351(6271):400-403.

5. Amantana A, Moulton HM, Cate ML, et al. Pharmacokinetics, biodistribution, stability and toxicity of a cell-penetrating peptide-morpholino oligomer conjugate. Bioconjug Chem. 2007;18(4):1325-1331.

6. Goemans NM, Tulinius M, van den Akker JT, et al. Systemic administration of PRO051 in Duchenne's muscular dystrophy. $N$ Engl J Med. 2011;364(16):1513-1522.

7. Kinali M, Arechavala-Gomeza V, Feng L, et al. Local restoration of dystrophin expression with the morpholino oligomer AVI-4658 in Duchenne muscular dystrophy: a single-blind, placebo-controlled, dose-escalation, proof-of-concept study. Lancet Neurol. 2009;8(10):918-928.

8. Koenig M, Beggs AH, Moyer M, et al. The molecular basis for Duchenne versus Becker muscular dystrophy: correlation of severity with type of deletion. Am J Hum Genet. 1989;45(4):498-506.

9. Lu QL, Mann CJ, Lou F, et al. Functional amounts of dystrophin produced by skipping the mutated exon in the mdx dystrophic mouse. Nat Med. 2003;9(8):1009-1014.

10. Lu QL, Rabinowitz A, Chen YC, et al. Systemic delivery of antisense oligoribonucleotide restores dystrophin expression in body-wide skeletal muscles. Proc Natl Acad Sci U S A. 2005;102(1):198-203.

11. Mendell JR, Rodino-Klapac LR, Sahenk Z, et al. Eteplirsen for the treatment of Duchenne muscular dystrophy. Ann Neurol. 2013;74(5): 637-647.

12. van Deutekom JC, Janson AA, Ginjaar IB, et al. Local dystrophin restoration with antisense oligonucleotide PRO051. N Engl J Med. 2007; 357(26):2677-2686.

13. Wu B, Moulton HM, Iversen PL, et al. Effective rescue of dystrophin improves cardiac function in dystrophin-deficient mice by a modified morpholino oligomer. Proc Natl Acad Sci U S A. 2008;105(39): 14814-14819.

14. Wu B, Li Y, Morcos PA, Doran TJ, Lu P, Lu QL. Octa-guanidine morpholino restores dystrophin expression in cardiac and skeletal muscles and ameliorates pathology in dystrophic mdx mice. Mol Ther. 2009; 17(5):864-871.

15. Wu B, Lu P, Cloer C, et al. Long-term rescue of dystrophin expression and improvement in muscle pathology and function in dystrophic mdx mice by peptide-conjugated morpholino. Am J Pathol. 2012;181(2):392-400. 
16. Yin H, Moulton HM, Seow Y, et al. Cell-penetrating peptide-conjugated antisense oligonucleotides restore systemic muscle and cardiac dystrophin expression and function. Hum Mol Genet. 2008;17(24): 3909-3918.

17. Eckstein F. Phosphorothioate oligodeoxynucleotides: what is their origin and what is unique about them? Antisense Nucleic Acid Drug Dev. 2000;10(2):117-121.

18. Koo T, Wood MJ. Clinical trials using antisense oligonucleotides in Duchenne muscular dystrophy. Hum Gene Ther. 2013;24(5): 479-488.

19. Hoffman EP. Skipping toward personalized molecular medicine. N Engl J Med. 2007;357(26):2719-2722.

20. 't Hoen PA, van der Wees CG, Aartsma-Rus A, et al. Gene expression profiling to monitor therapeutic and adverse effects of antisense therapies for Duchenne muscular dystrophy. Pharmacogenomics. 2006;7(3):281-297.

21. Sirsi SR, Schray RC, Guan X, et al. Functionalized PEG-PEI copolymers complexed to exon-skipping oligonucleotides improve dystrophin expression in mdx mice. Hum Gene Ther. 2008;19(8):795-806.

22. Williams JH, Sirsi SR, Latta DR, Lutz GJ. Induction of dystrophin expression by exon skipping in $m d x$ mice following intramuscular injection of antisense oligonucleotides complexed with PEG-PEI copolymers. Mol Ther. 2006;14(1):88-96.

23. Alshamsan A, Haddadi A, Incani V, Samuel J, Lavasanifar A, Uludağ H. Formulation and delivery of siRNA by oleic acid and stearic acid modified polyethylenimine. Mol Pharm. 2009;6(1):121-133.

24. Huang Y, Rao Y, Chen J, Yang VC, Liang W. Polysorbate cationic synthetic vesicle for gene delivery. $J$ Biomed Mater Res A. 2011;96(3):513-519.

25. Wang M, Wu B, Lu P, Cloer C, Tucker JD, Lu Q. Polyethyleniminemodified pluronics (PCMs) improve morpholino oligomer delivery in cell culture and dystrophic $m d x$ mice. Mol Ther. 2013;21(1):210-216.

26. Wang M, Wu B, Lu P, et al. Pluronic-PEI copolymers enhance exonskipping of 2'-O-methyl phosphorothioate oligonucleotide in cell culture and dystrophic $m d x$ mice. Gene Ther. 2014;21(1):52-59.

27. Wang M, Wu B, Tucker JD, Lu P, Bollinger LE, Lu Q. Tween 85 grafted PEIs enhanced delivery of antisense 2'-O-methyl phosphorothioate oligonucleotides in vitro and in dystrophic mdx mice. J Mater Chem B. 2015;3(26):5330-5340.

28. Jirka SMG, 't Hoen PAC, Diaz Parillas V, et al. Cyclic Peptides to Improve Delivery and Exon Skipping of Antisense Oligonucleotides in a Mouse Model for Duchenne Muscular Dystrophy. Mol Ther. 2018;26(1):132-147.

29. Jirka SM, Heemskerk H, Tanganyika-de Winter CL, et al. Peptide conjugation of 2'-O-methyl phosphorothioate antisense oligonucleotides enhances cardiac uptake and exon skipping in mdx mice. Nucleic Acid Ther. 2014;24(1):25-36.

30. Kendall GC, Mokhonova EI, Moran M, et al. Dantrolene enhances antisense-mediated exon skipping in human and mouse models of Duchenne muscular dystrophy. Sci Transl Med. 2012;4(164):ra160.

31. Cao L, Han G, Lin C, et al. Fructose Promotes Uptake and Activity of Oligonucleotides With Different Chemistries in a Context-dependent Manner in mdx Mice. Mol Ther Nucleic Acids. 2016;5(6):e329.
32. Han G, Gu B, Cao L, et al. Hexose enhances oligonucleotide delivery and exon skipping in dystrophin-deficient mdx mice. Nat Commun. 2016;7:10981

33. Wang M, Wu B, Shah SN, Lu P, Lu Q. Saponins as Natural Adjuvant for Antisense Morpholino Oligonucleotides Delivery In Vitro and in mdx Mice. Mol Ther Nucleic Acids. 2018;11:192-202.

34. Ferreira F, Llodra J. Glycoside based adjuvants. In: Oleszek W, Marston A, editors. Saponins in Food, Feedstuffs and Medicinal Plants. The Netherlands: Kluwer Academic Publishers; 2000:233-240.

35. Rajput ZI, Hu SH, Xiao CW, Arijo AG. Adjuvant effects of saponins on animal immune responses. J Zhejiang Univ Sci B. 2007;8(3): $153-161$.

36. Wang Y, Wang X, Huang J, Li J. Adjuvant Effect of Quillaja saponaria Saponin (QSS) on Protective Efficacy and IgM Generation in Turbot (Scophthalmus maximus) upon Immersion Vaccination. Int J Mol Sci. 2016;17(3):325.

37. Fiskum G. Intracellular levels and distribution of $\mathrm{Ca} 2+$ in digitoninpermeabilized cells. Cell Calcium. 1985;6(1-2):25-37.

38. Geelen MJ. The use of digitonin-permeabilized mammalian cells for measuring enzyme activities in the course of studies on lipid metabolism. Anal Biochem. 2005;347(1):1-9.

39. Arase Y, Ikeda K, Murashima N, et al. The long term efficacy of glycyrrhizin in chronic hepatitis C patients. Cancer. 1997;79(8): 1494-1500.

40. van Rossum TG, Vulto AG, de Man RA, Brouwer JT, Schalm SW. Review article: glycyrrhizin as a potential treatment for chronic hepatitis C. Aliment Pharmacol Ther. 1998;12(3):199-205.

41. Cartwright AC. The British Pharmacopoeia, 1864 to 2014: Medicines, International Standards and the State. Vol. 183. UK: Routledge; 2016.

42. Hollman A. Drugs for atrial fibrillation. Digoxin comes from Digitalis lanata. BMJ. 1996;312(7035):912.

43. Friedman M. Anticarcinogenic, cardioprotective, and other health benefits of tomato compounds lycopene, $\alpha$-tomatine, and tomatidine in pure form and in fresh and processed tomatoes. J Agric Food Chem. 2013;61(40):9534-9550.

44. Dyle MC, Ebert SM, Cook DP, et al. Systems-based discovery of tomatidine as a natural small molecule inhibitor of skeletal muscle atrophy. J Biol Chem. 2014;289(21):14913-14924.

45. Griffin WC. Classification of Surface-Active Agents by 'HLB'. J Soc Cosmet Chem. 1949;1(5):311-326.

46. Griffin WC. Calculation of HLB Values of Non-Ionic Surfactants J Soc Cosmet Chem. 1954;5(4):249-256.

47. $\mathrm{Hu}$ Y, Wu B, Zillmer A, et al. Guanine analogues enhance antisense oligonucleotide-induced exon skipping in dystrophin gene in vitro and in vivo. Mol Ther. 2010;18(4):812-818.

48. Sazani P, Kang SH, Maier MA, et al. Nuclear antisense effects of neutral, anionic and cationic oligonucleotide analogs. Nucleic Acids Res. 2001;29(19):3965-3974.

\section{Publish your work in this journal}

Drug Design, Development and Therapy is an international, peerreviewed open-access journal that spans the spectrum of drug design and development through to clinical applications. Clinical outcomes, patient safety, and programs for the development and effective, safe, and sustained use of medicines are the features of the journal, which

\section{Dovepress}

has also been accepted for indexing on PubMed Central. The manuscript management system is completely online and includes a very quick and fair peer-review system, which is all easy to use. Visit http://www.dovepress.com/testimonials.php to read real quotes from published authors. 